The Astrophysical Journal, 476:L77-L80, 1997 February 20

(C) 1997. The American Astronomical Society. All rights reserved. Printed in U.S.A.

\title{
EVIDENCE FOR AN X-RAY-EMITTING GALACTIC BULGE: SHADOWS CAST BY DISTANT MOLECULAR GAS
}

\author{
SAngwook Park and John P. Finley \\ Department of Physics, Purdue University 1396 Physics Building, West Lafayette, IN 47907; \\ parksan@purds1.physics.purdue.edu, finley@purds1.physics.purdue.edu \\ S. L. SNOWDEN ${ }^{1}$ \\ NASA/Goddard Space Flight Center, Code 662, Greenbelt, MD 20771; snowden@lheavx.gsfc.nasa.gov \\ AND \\ T. M. DAME \\ Harvard-Smithsonian Center for Astrophysics, 60 Garden Street, Cambridge, MA 02138; tdame@cfa.harvard.edu \\ Received 1996 July 11; accepted 1996 December 3
}

\begin{abstract}
A mosaic of seven ROSAT PSPC pointed observations in the direction of $\left(l, b \sim 10^{\circ}, 0^{\circ}\right)$ reveals deep X-ray shadows in the $0.5-2.0 \mathrm{keV}$ band cast by dense molecular gas. The comparison between the observed on-cloud and off-cloud X-ray fluxes indicates that $\sim 43 \%$ of the diffuse X-ray background in this direction in both the 0.75 and $1.5 \mathrm{keV}$ bands originates behind the molecular gas, which is located at 2-4 kpc from the Sun. Given the short mean free path of X-rays in the $0.75 \mathrm{keV}$ band in the Galactic plane ( $\sim 1 \mathrm{kpc}$ assuming an average space density of $\left.1 \mathrm{~cm}^{-3}\right)$, this large percentage of the observed flux that originates beyond the molecular gas most likely indicates a strong enhancement in the distribution of X-ray-emitting gas in the Galactic center region, possibly associated with a Galactic X-ray bulge.
\end{abstract}

Subject headings: Galaxy: structure - ISM: structure - X-rays: galaxies — X-rays: ISM

\section{INTRODUCTION}

The origin of the $\sim 0.5-2.0 \mathrm{keV}$ diffuse $\mathrm{X}$-ray background has long eluded a satisfactory explanation. The extrapolation of the extragalactic power law $\left(I_{\mathrm{X}} \sim 11 E^{-1.4}\right.$ photons $\mathrm{cm}^{-2} \mathrm{~s}^{-1}$ $\mathrm{sr}^{-1} \mathrm{keV}^{-1}$ ) from the 2-10 keV band (Henry et al. 1971; Bunner, Sanders, \& Nousek 1979) accounts for $\lesssim 50 \%$ of the observed X-ray intensity at high Galactic latitudes in the $\sim 0.5-1.0 \mathrm{keV}$ band (McCammon et al. 1983). While there remains some discussion as to the exact normalization of the power law and shape of the spectrum below $2 \mathrm{keV}$ (e.g., $9.2 E^{-1.4}$ and no appreciable excess between 1 and $2 \mathrm{keV}$, Gendreau 1995; 10.8E $E^{-1.4}$, Chen, Fabian, \& Gendreau 1996), all measurements agree that there is a strong excess above extrapolation of the $2-10 \mathrm{keV}$ power-law spectrum in the $0.5-1.0 \mathrm{keV}$ band. An upper limit to the contribution from the local hot bubble (LHB) (Snowden et al. 1990), which extends out from 50 to 200 parsecs, is $\sim 20 \%$ of the nominal highlatitude intensity, as revealed by a high-latitude X-ray shadow due to a nearby molecular cloud MBM 12 (Snowden, McCammon, \& Verter 1993). The contributions from stellar sources (e.g., late-type stars) have been considered to explain the observed excess X-ray intensity in the Galactic plane (Rosner et al. 1981; Schmitt \& Snowden 1990; Wang 1992; Ottmann \& Schmitt 1992); however, they do not produce a sufficient flux. Although a substantial fraction of the thermal excess of the diffuse X-ray background at high Galactic latitudes is from discrete extragalactic sources (e.g., AGNs), at least $25 \%$ of the diffuse X-ray emission is not caused by AGNs (Hasinger et al. 1993). A significant portion of the diffuse X-rays could still be Galactic, yet from beyond the LHB even at high latitudes. The problem is even more severe when the intensity near the Galactic plane is considered. The Galactic plane is optically

\footnotetext{
${ }^{1}$ Universities Space Research Association.
}

thick at these energies, so all of the observed flux must be Galactic in origin.

The Galactic diffuse X-ray emission beyond the LHB strongly suggests an abundance of Galactic gas at $\sim 3 \times 10^{6} \mathrm{~K}$ (Sanders et al. 1982). The distribution of the X-ray-emitting material in the Galactic plane beyond obvious supernova remnants and stellar wind bubbles is, however, poorly known. Snowden et al. (1996) have recently suggested that the strong $\mathrm{X}$-ray intensity seen above and below the plane in the general direction of the Galactic center is the result of a Galactic X-ray bulge. The best way to study the Galactic distribution of the $\mathrm{X}$-ray-emitting gas is to search for shadows in the diffuse $\mathrm{X}$-ray background cast by absorbing high column density molecular gas for which the distance can be determined. The utility of this approach has been demonstrated in the literature in the case of nearby neutral and molecular clouds at high Galactic latitudes (e.g., Burrows \& Mendenhall 1991; Snowden et al. 1991; Snowden, McCammon, \& Verter 1993; Wang \& Yu 1995; Kuntz, Snowden, \& Verter 1996). Here we report the detection of deep X-ray shadows cast by dense molecular gas in the inner Galaxy. These shadows imply an extensive flux of diffuse X-rays from the Galactic center region.

The line of sight of our study region $\left(l, b \sim 10^{\circ}, 0^{\circ}\right)$ extensively samples the inner Galaxy $(1.5 \mathrm{kpc}$ minimum radius), but still allows reliable velocity-derived distances for the molecular gas. This region has been covered by a mosaic of seven independent ROSAT PSPC pointed observations, and by the Galactic CO survey of Bitran (1987). We use the CO to derive both kinematic distances and molecular column densities for the absorbing gas and the IRAS $100 \mu \mathrm{m}$ intensity as a tracer of total gas column density.

The data used for this study are described in $\S 2$. The analysis and the implications of the results are discussed in $\S 3$, and a summary and some conclusions are presented in $\S 4$. 
TABLE 1

LIST OF ROSAT PSPC OBSERVATIONS

\begin{tabular}{|c|c|c|c|c|}
\hline Observation ID & $l$ & $b$ & $\begin{array}{l}\text { Exposure } \\
\text { (ks) }\end{array}$ & Date \\
\hline WP500197N00 & 8.76 & -0.11 & 3.14 & 1992 Oct 5 \\
\hline WP200717 ............ & 10.80 & 0.39 & 8.06 & 1992 Mar 7-8 \\
\hline RP500150N00........... & 11.12 & -0.35 & 4.87 & 1993 Mar 4 \\
\hline WP900145 ........ & 9.96 & -0.24 & 2.44 & 1991 Mar $18-27$ \\
\hline WP900399N00 .......... & 9.96 & -0.24 & 9.39 & 1993 Apr 2-3 \\
\hline WP180034N00 .......... & 9.71 & -0.39 & 1.38 & 1993 Oct $9-10$ \\
\hline RP201060N00......... & 9.24 & -0.61 & 8.50 & 1993 Apr 2-3 \\
\hline
\end{tabular}

\section{DATA}

The CO data used in the present study were taken from the Galactic survey of Bitran (1987), which was carried out with the $1.2 \mathrm{~m}$ millimeter-wave telescope at Cerro Tololo, Chile. At $115 \mathrm{GHz}$, the frequency of the $J=1-0$ rotational transition, this telescope has a beamwidth of 8:8 (FWHM) and its 256-channel spectrometer provides a velocity resolution of 1.3 $\mathrm{km} \mathrm{s}^{-1}$ and total bandwidth of $333 \mathrm{~km} \mathrm{~s}^{-1}$. Observations were spaced roughly every beamwidth (7.5) on a Galactic grid, and the rms sensitivity of $0.14 \mathrm{~K}\left(T_{\mathrm{mb}}\right)$ was more than adequate for the present purpose.

The IRAS $100 \mu \mathrm{m}$ data (Wheelock et al. 1994) for this region were obtained through the High Energy Astrophysics Science Archive (HEASARC) SkyView facility, which is maintained by NASA/GSFC. The displayed IRAS $100 \mu \mathrm{m}$ field (Fig. 1 [Pl. L6, L7]) is $4.3 \times 4.3$ centered at $(l, b=9: 84$, $-0.15)$. The infrared intensity for this field ranges from $\sim 200$ $\mathrm{MJy} \mathrm{sr}{ }^{-1}$ to $10^{4} \mathrm{MJy} \mathrm{sr}^{-1}$, and the angular resolution is $\sim 5^{\prime}$.

The seven ROSAT (Trümper 1992) PSPC pointed observations are listed in Table 1. Data from all seven pointings were obtained through the HEASARC ROSAT public archive. Since noncosmic contamination is not negligible in the study of the diffuse X-ray background, all identified noncosmic contributions to the counting rate must be handled thoroughly. The particle background, scattered solar X-ray background, and long-term enhancements are modeled and subtracted from the data by following the methods described in the literature (Snowden et al. 1994, and references therein). Contamination caused by short-term enhancements including auroral X-rays, solar flares, and enhanced charged-particle rates encountered near the South Atlantic Anomaly and particle belts are removed by excluding all observation time intervals that display anomalous peaks in their light curves. For these seven pointings, the modeled noncosmic contamination ranges up to $\sim 50 \%$ of the total observed X-ray counts, and the mean contribution is $\sim 20 \%$.

The individual PSPC pointings with all identified noncosmic contamination, point sources, and discrete small-scale extended emission features (e.g., G11.2-0.3 and W30) removed are merged into large area mosaics in two bands: the $0.44-1.21$ $\mathrm{keV}$ band $(0.75 \mathrm{keV})$ and the $0.73-2.04 \mathrm{keV}$ band $(1.5 \mathrm{keV})$. The large amount of spectral overlap between the two bands is a result of the relatively poor spectral resolution of the proportional counter. The determination of the relative offsets in the zero level between the individual observations is performed by comparing the average count rates in the overlapping regions between all pairs of observations. The contribution from this correction is typically small, and is $\lesssim 3 \%$ of the total counts for this field of view. The software for this task (Snowden 1994) was provided by the US ROSAT Science
Data Center (USRSDC) at NASA/GSFC. The feasibility of the software has been successfully demonstrated by a mosaic of the Large Magellanic Cloud (Snowden \& Petre 1994).

The final ROSAT PSPC mosaics of the region $\left(l, b \sim 10^{\circ}\right.$, $0^{\circ}$ ) are displayed in Figures $1 a$ and $1 b$. For purposes of display, the data were binned into $5^{\prime}$ pixels and smoothed. For the analysis of this paper, the unsmoothed $5^{\prime}$ pixels were used, usually with further binning. This pixel size was selected since it is reasonably comparable to the spatial resolution of the IRAS $100 \mu \mathrm{m}$ and the $\mathrm{CO}$ data, without losing most of the detailed spatial structure of the X-ray emission. Combined as a mosaic, the average exposure for this field is $\sim 18 \mathrm{ks}$, yielding $\sim 15 \%$ statistical errors for individual pixels.

\section{ANALYSIS AND DISCUSSION}

The Galactic plane in the range $l \sim 10^{\circ}-11^{\circ}$ shows a broad maximum in the far infrared and a corresponding minimum in $0.5-2.0 \mathrm{keV}$ band X-rays (Figs. $1 a$ and $1 b$ ). Although the intense pointlike features in the far-infrared map (Fig. 1) arise from well-known Galactic H II regions such as W31 (the cluster of sources near the center of the map), the more extended emission is considered to be a fairly reliable tracer of total gas column density on a Galactic scale (Bloemen, Deul, \& Thaddeus 1990). No significant CO concentration is associated with any of the IRAS cores in all velocity intervals except for the one associated with SGR 1806-20 at $15 \mathrm{kpc}$ (Fig. 2a, Corbel et al. 1996). Since the in-plane gas density peaks about half-way between the Sun and the Galactic center (Dame 1993), the anticorrelation seen in Figure 1 suggests that a significant fraction of the X-rays arise from beyond the bulk of the gas, most likely in the Galactic bulge.

This location of the bulk of the X-ray-emitting gas is supported by the more detailed anticorrelation seen between $\mathrm{X}$-rays and the dense molecular component of the gas as traced by $\mathrm{CO}$. In Figure 2, the $\mathrm{CO}$ emission is partitioned into four equal intervals of velocity, and overlaid as contours on the $1.5 \mathrm{keV}$ band X-ray map that has been smoothed to match the angular resolution of the $\mathrm{CO}$ data. These four maps account for nearly all of the $\mathrm{CO}$ emission in this direction; similar $\mathrm{CO}$ maps at higher velocities are blank or nearly so. The $\mathrm{CO}$ emission is strongest and most widespread in the velocity range $15-30 \mathrm{~km} \mathrm{~s}^{-1}$ (Fig. $2 b$ ), and it is this emission that shows the best detailed anticorrelation with the X-rays. According to the Galactic rotation curve of Burton (1988), such emission must arise either from the near distance interval 2.5-3.9 kpc (as indicated above the plot) or from the corresponding far interval $12.8-14.3 \mathrm{kpc}$. With such a large difference between the near and far distances, $\mathrm{CO}$ maps at the scale and resolution of Figure 2 would tend to be dominated by near side material (given the typical scale height), and the anticorrelation with the X-rays implies that such is probably the case here.

Figure $2 b$ displays apparent $\mathrm{X}$-ray shadows cast by molecular gas at different velocities and distances. The Y-shaped shadow extending above the plane near $l \sim 10.5$ is perhaps the most notable, both because of its detailed match to the $\mathrm{CO}$, and the relatively high velocity $\left(\sim 30 \mathrm{~km} \mathrm{~s}^{-1}\right)$ and distance $(3.9 \pm 0.7 \mathrm{kpc})$ of the absorbing gas; this same vertical structure is even partially seen in the $30-45 \mathrm{~km} \mathrm{~s}^{-1}$ map (Fig. 2c). The clarity of this shadow might be due in part to its relatively high latitude, where confusion by background material at the far kinematic distance should be negligible. Two other welldefined shadows are seen near $l \sim 8 \% 6$, and are also well 

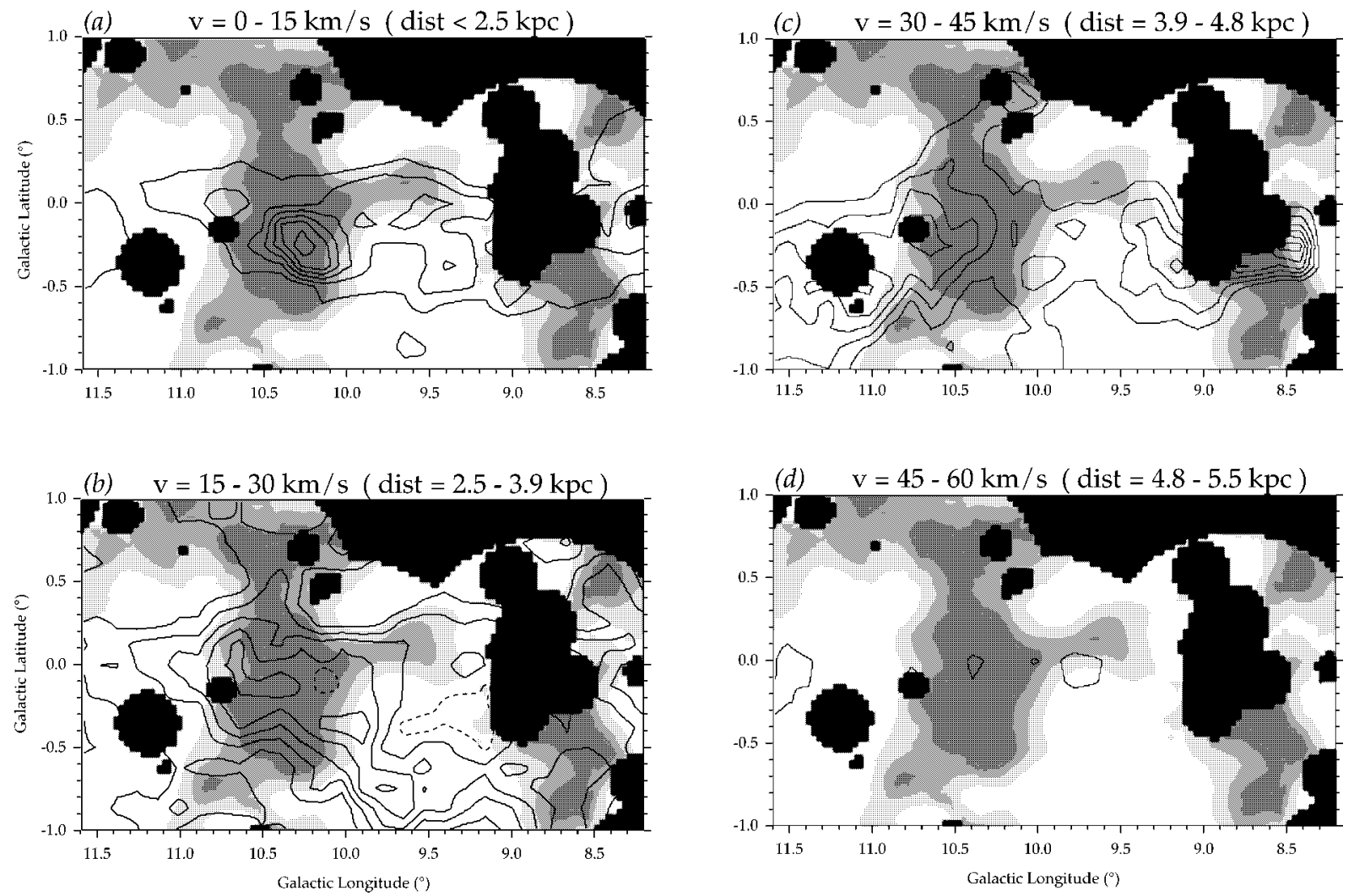

FIG. 2.- ${ }^{12} \mathrm{CO}(J=1-0)$ intensity in four different velocity intervals overlaid on the gray-scale image of the $1.5 \mathrm{keV}$ band X-rays that has been smoothed to match the 8.8 angular resolution of the $\mathrm{CO}$. The $\mathrm{CO}$ contours are at $28,38,48,58$, and $68 \mathrm{~K} \mathrm{~km} \mathrm{~s}^{-1}$. The contour at $28 \mathrm{~K} \mathrm{~km} \mathrm{~s}^{-1}$ corresponds to $\sim 2$ optical depths in the $0.75 \mathrm{keV}$ band and $\sim 1.4$ optical depths in the $1.5 \mathrm{keV}$ band. The $\mathrm{CO}$ velocity integration range is given above each plot, along with the corresponding range of the near kinematic distances, according to the Galactic rotation curve of Burton (1988). The X-ray gray scale is strongly saturated to white at high intensities in order to emphasize the regions of absorption.

displaced from the plane: one at $b \sim 0.5$ and the other at $b \sim$ -0 .7. Both features have gas velocities near $18 \mathrm{~km} \mathrm{~s}^{-1}$, corresponding to a near kinematic distance of $3.1 \pm 0.5 \mathrm{kpc}$. The most notable exception to the general anticorrelation seen in Figure 2 is the region near $(l, b \sim 11.25,-0.5)$, which is very bright in X-rays yet shows relatively strong $\mathrm{CO}$ emission at distances greater than $3.9 \mathrm{kpc}$ (Fig. 2c). The bulk of these $\mathrm{X}$-rays most likely arise from a foreground diffuse X-ray emission feature.

In the regions where the absorption is clear- $\left(l, b \sim 10^{\circ} .3\right.$, $-0.2),(l, b \sim 8.6,-0.7)$, and $(l, b \sim 10.5,0.5)$-(see Fig. $2 b)$, the $\mathrm{H}_{2}$ column density for the molecular gas is estimated to be $\gtrsim 1 \times 10^{22} \mathrm{~cm}^{-2}$ by applying a $\mathrm{CO}-\mathrm{H}_{2}$ conversion factor $\mathrm{H}_{2} / \mathrm{CO}=2 \times 10^{20} \mathrm{~cm}^{-3}\left(\mathrm{~K} \mathrm{~km} \mathrm{~s}^{-1}\right)^{-1}$. This large molecular column density indicates that the molecular gas in these directions is optically thick for the $0.5-2.0 \mathrm{keV} \mathrm{X}$-rays (i.e., one optical depth is $\sim 2.7 \times 10^{21} \mathrm{~cm}^{-2}$ in the $0.75 \mathrm{keV}$ band, and $\sim 4 \times 10^{21} \mathrm{~cm}^{-2}$ in the $1.5 \mathrm{keV}$ band, assuming the theoretical cross section of Morrison \& McCammon 1983 and a $10^{6.6} \mathrm{~K}$ thermal plasma). Therefore, it is possible to directly extract the on-cloud and off-cloud X-ray intensities in order to estimate the foreground and distant fractions of the observed X-ray flux rather than fitting the standard two-component absorption model (e.g., Snowden et al. 1993).

The on-cloud X-ray emission is derived from a $1200 \mathrm{arcmin}^{2}$ extraction region centered on $(l, b \sim 10.3,-0.2)$, a 600 $\operatorname{arcmin}^{2}$ extraction region centered on $(l, b \sim 8.6,-0.7)$, and a $300 \operatorname{arcmin}^{2}$ extraction region centered on $(l, b \sim 10.5,0.5)$.
The range of on-cloud X-ray intensity in the $0.75 \mathrm{keV}$ band from these three regions is $(53-57) \times 10^{-6}$ counts $\mathrm{s}^{-1}$ $\operatorname{arcmin}^{-2}$, while the $1.5 \mathrm{keV}$ band intensity is $\sim 94 \times 10^{-6}$ counts $\mathrm{s}^{-1} \operatorname{arcmin}^{-2}$ in all three regions. Several regions with $I_{\mathrm{CO}}<28 \mathrm{~K} \mathrm{~km} \mathrm{~s}^{-1}$ (i.e., $\mathrm{H}_{2}$ density $\lesssim 5 \times 10^{21} \mathrm{~cm}^{-2}$ ) were selected to estimate the off-cloud $X$-ray intensities in the field: a $750 \operatorname{arcmin}^{2}$ region centered on $(l, b \sim 9.9,0.4)$, a 400 $\operatorname{arcmin}^{2}$ region centered on $(l, b \sim 11.3,0.5)$, a $600 \mathrm{arcmin}^{2}$ region centered on $\left(l, b \sim 11^{\circ} 0,-1.1\right)$, and a $375 \operatorname{arcmin}^{2}$ region centered on $\left(l, b \sim 9^{\circ} 0,-1^{\circ} .3\right)$. The brightest regionsi.e., $(l, b \sim 11.5,-0.5)$ and $(l, b \sim 9.3,-1.0)$-were excluded to avoid contamination by possible discrete X-ray emission features. The off-cloud X-ray intensities, the sum of foreground and distant emission, lie in the range $(\sim 87-109) \times 10^{-6}$ counts $\mathrm{s}^{-1} \operatorname{arcmin}^{-2}$ in the $0.75 \mathrm{keV}$ band and $(\sim 149-179) \times 10^{-6}$ counts $\mathrm{s}^{-1} \operatorname{arcmin}^{-2}$ in the $1.5 \mathrm{keV}$ band. The observed on-cloud and average off-cloud X-ray intensities for this field of view are summarized in Table 2. The off-cloud X-ray intensities are consistent with the range of $0.5-2.0 \mathrm{keV} R O S A T$ all-sky survey values (Snowden et al. 1995) in the first quadrant of the Galactic plane.

The average on-cloud to off-cloud X-ray intensity ratio in the $0.75 \mathrm{keV}$ band is $0.57 \pm 0.09$, which implies that $43 \% \pm$ $9 \%$ of the observed X-ray flux in this band originates behind the molecular cloud. Given the probable large X-ray absorption optical depth foreground to the cloud region $(\tau \sim 3.4$, assuming an average space density of $\sim 1 \mathrm{H} \mathrm{I} \mathrm{cm}^{-3}$ in the midplane [Slavin \& Cox 1992] and a distance of $\sim 3 \mathrm{kpc}$ ), a 
TABLE 2

Observed On-Cloud and OfF-Cloud X-Ray FluXes

\begin{tabular}{ccrc}
\hline \hline $\begin{array}{c}\text { Band } \\
(\mathrm{keV})\end{array}$ & On-Cloud $^{\mathrm{a}}$ & Off-Cloud $^{\mathrm{a}}$ & Ratio \\
\hline $0.75 \ldots \ldots \ldots$ & $55 \pm 6$ & $96 \pm 10$ & $0.57 \pm 0.09$ \\
$1.5 \ldots \ldots \ldots$. & $94 \pm 9$ & $168 \pm 17$ & $0.56 \pm 0.08$ \\
\hline
\end{tabular}

${ }^{a}$ On-cloud and off-cloud fluxes in units of $10^{-6}$ counts $\mathrm{s}^{-1}$ $\operatorname{arcmin}^{-2}$. The errors include both the formal statistical uncertainty and a systematic contribution related to the selection of the on-cloud and off-cloud regions.

bright background emission region is implied: $\sim 1229 \times 10^{-6}$ counts $\mathrm{s}^{-1} \operatorname{arcmin}^{-2}$, even assuming no additional absorption between the cloud and the distant emission region. This value is roughly an order of magnitude greater than the nominal highlatitude intensity $\left(\sim 130 \times 10^{-6}\right.$ counts $\left.\mathrm{s}^{-1} \operatorname{arcmin}^{-2}\right)$. This emission is consistent with the extrapolation to the plane of the high $\mathrm{X}$-ray intensities seen above and below the plane (interpreted as a Galactic X-ray bulge by Snowden et al. 1996) in this direction.

The average on-cloud to off-cloud X-ray flux ratio in the $1.5 \mathrm{keV}$ band of $0.56 \pm 0.08$ is nearly the same as that for the $0.75 \mathrm{keV}$ band, and the foreground optical depth is $\tau \sim 2.3$ (assuming 1 optical depth $\sim 4 \times 10^{21} \mathrm{~cm}^{-2}$ ). The interpretation is the same as for the $0.75 \mathrm{keV}$ band shadow: there is a strong enhancement in the filling factor of X-ray-emitting gas in the Galactic plane beyond $\sim 3 \mathrm{kpc}$ in the direction of the Galactic center. The implied background emission, again with no additional absorption, is $\sim 725 \times 10^{-6}$ counts $\mathrm{s}^{-1} \operatorname{arcmin}^{-2}$. The de-absorbed $1.5 / 0.75 \mathrm{keV}$ band ratio for the distant emission, $\sim 0.59$, suggests a thermal emission temperature of $10^{6.7} \mathrm{~K}$. This is again consistent with the Galactic X-ray bulge of Snowden et al. (1996).

\section{SUMMARY AND CONCLUSIONS}

We have presented and discussed the implications of the deep $0.5-2.0 \mathrm{keV}$ band X-ray shadows in the Galactic plane cast by dense inner Galaxy molecular gas toward $l \sim 10^{\circ}$. The striking result that one-half of the observed intensity originates beyond an optical depth of 2.3-3.4 requires that the emitting gas is nonuniformly distributed in the Galactic disk and has a strong enhancement near the Galactic center. Its existence is also consistent with the extrapolation to the plane of the emission from a probable Galactic X-ray bulge (Snowden et al. 1996).

While some of the emission responsible for the foreground component could arise in the nearby $(\sim 150 \mathrm{pc}$ with a $\sim 100 \mathrm{pc}$ radius) Sco-Cen bubble, a part of this emission in all likelihood originates from another source component or superposition of multiple components that may be distributed over the $\sim 3 \mathrm{kpc}$ to the molecular gas. The additional component(s) presumably contributes also to the observed nonzero flux along the Galactic plane, observed in the anticenter direction where any extragalactic emission is still completely absorbed. Further analysis of additional shadowing targets will help clarify the situation.

The authors would like to thank J. Lockman for helpful discussion and direction, and the referee, D. McCammon, for his thoughtful review of this paper. This research has made use of data obtained through the High Energy Astrophysics Science Archive Research Center Online Service, provided by the NASA/Goddard Space Flight Center, and it was supported in part by NASA grant NAG 5-2492 and the Purdue Research Foundation.

\section{REFERENCES}

Bitran, M. E. 1987, Ph.D. thesis, Univ. Florida

Bloemen, J. B. G. M., Deul, E. R., \& Thaddeus, P. 1990, A\&A, 233, 437

Bunner, A. N., Sanders, W. T., \& Nousek, J. A. 1979, ApJ, 228, L29

Burrows, D. N., \& Mendenhall, J. A. 1991, Nature, 351, 629

Burton, W. B. 1988, in Galactic and Extra-Galactic Radio Astronomy, ed. G. L.

Verschuur \& K. I. Kellermann (Berlin: Springer), 295

Chen, L.-W., Fabian, A. C., \& Gendreau, K. C. 1996, MNRAS, submitted

Corbel, S., Wallyn, P., Dame, T. M., Durouchoux, P., Mahoney, W. A., Vilhu,

O., \& Grindlay, J. E. 1996, ApJ, in press

Dame, T. M. 1993, in AIP Conf. Proc. 278, Back to the Galaxy, ed. S. Holt \&

F. Verter (San Francisco: AIP), 267

Gendreau, K. C. 1995, Ph.D. thesis, MIT

Hasinger, G., Burg, R., Giacconi, R., Hartner, G., Schmidt, M., Trümper, J., \& Zamorani, G. 1993, A\&A, 275, 1

Henry, R. C., Fritz, G., Meekins, J. F., Chubb, T., \& Friedman, H. 1971, ApJ, 163, L73

Kuntz, K. D., Snowden, S. L., \& Verter, F. 1996, ApJ, submitted

McCammon, D., Burrows, D. N., Sanders, W. T., \& Kraushaar, W. L. 1983, ApJ, 269, 107

Morrison, R., \& McCammon, D. 1983, ApJ, 270, 119

Ottmann, R., \& Schmitt, J. H. M. M. 1992, A\&A, 256, 421

Rosner, R., et al. 1981, ApJ, 249, L5

Sanders, W. T., Burrows, D. N., Kraushaar, W. L., \& McCammon, D. 1982, in

IAU Symp. 101, Supernova Remnants and their X-Ray Emission, ed. J.

Danziger \& P. Gorenstein (Dordrecht: Reidel)
Schmitt, J. H. M. M., \& Snowden, S. L. 1990, ApJ, 361, 207

Slavin, J. D., \& Cox, D. P. 1992, ApJ, 392, 131

Snowden, S. L. 1994, Cookbook for Analysis Procedures for ROSAT XRT/PSPC Observations of Extended Sources and the Diffuse Background, HEASARC/USRSDC, GSFC

Snowden, S. L., Cox, D. P., McCammon, D., \& Sanders, W. T. 1990, ApJ, 354, 211

Snowden, S. L., et al. 1995, ApJ, 454, 643

Snowden, S. L., Freyberg, M. J., Schmitt, J. H. M. M., Voges, W., Trümper, J.,

McCammon, D., Plucinsky, P. P., \& Sanders, W. T. 1996, ApJ, submitted

Snowden, S. L., McCammon, D., Burrows, D. N., \& Mendenhall, J. A. 1994, ApJ, 424, 714

Snowden, S. L., McCammon, D., \& Verter, F. 1993, ApJ, 409, L21

Snowden, S. L., Mebold, U., Hirth, W., Herbstmeier, U., \& Schmitt, J. H.

M. M. 1991, Science, 252, 1529

Snowden, S. L. \& Petre, R. 1994, ApJ, 436, L123

Trümper, J. 1992, QJRAS, 33, 165

Wang, Q. D. 1992, ApJ, 392, 509

Wang, Q. D., \& Yu, K. C. 1995, AJ, 109, 698

Wheelock, S. L., et al. 1994, IRAS Sky Survey Atlas Explanatory Supplement (Pasadena: JPL), 94-11 


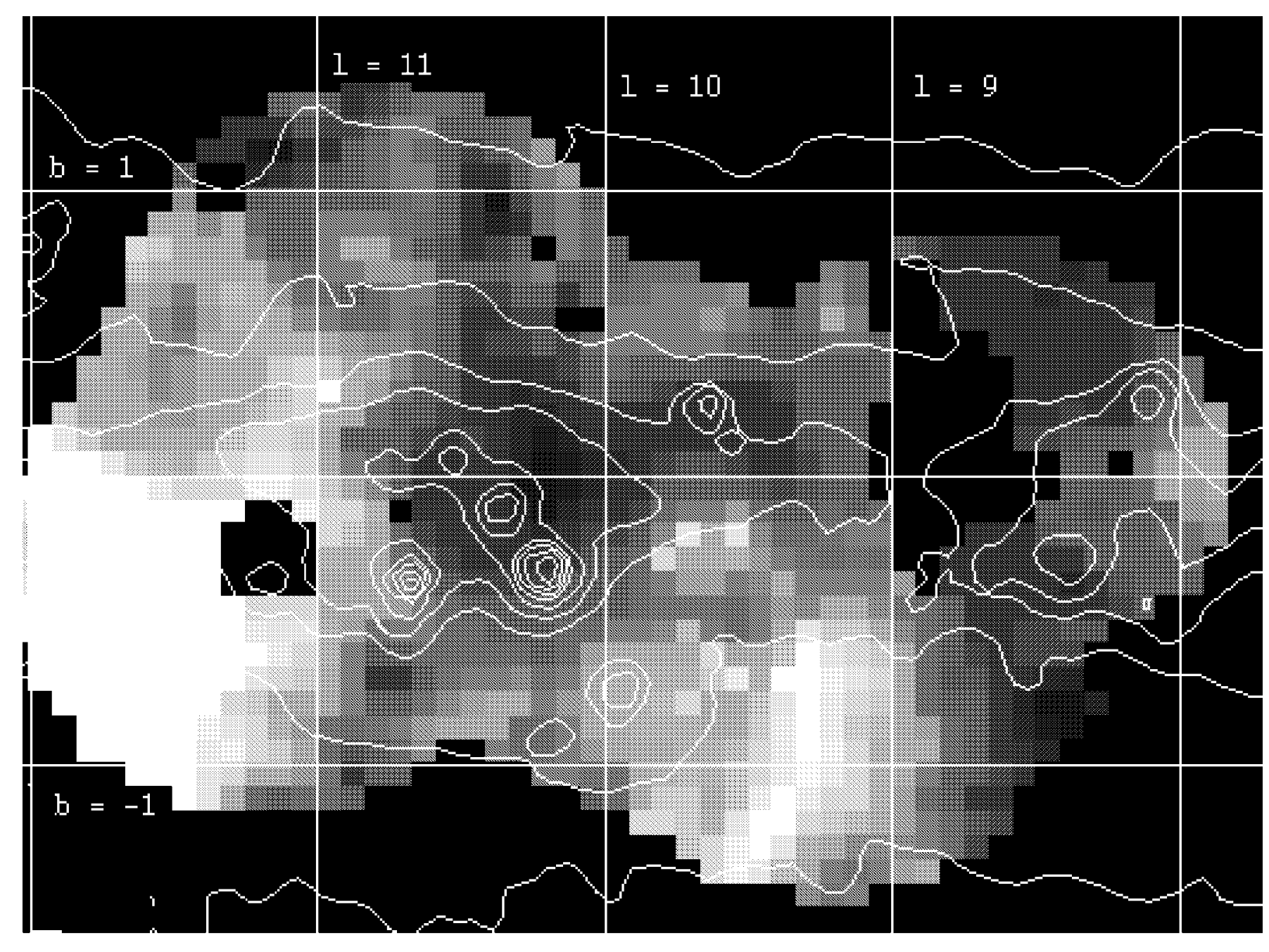

FIG, $1 a$

Fig. 1.-X-ray images of the Galactic plane near $l \sim 10^{\circ}$. These cover an $\sim 14 \mathrm{deg}^{2}$ region between $l=7.5$ and $l=12^{\circ}$, and $|b| \leq 1.5$, including the W30 complex, the W31 $\mathrm{H}$ II region, and SNR G11.2-0.3. The pixel size is $5^{\prime} \times 5^{\prime}$. Panels $(a)$ and $(b)$ display the 0.75 and the $1.5 \mathrm{keV}$ X-ray images, respectively, from the mosaic of the seven ROSAT PSPC pointed observations in Table 1 overlaid with the IRAS $100 \mu \mathrm{m}$ contours. The IRAS $100 \mu \mathrm{m}$ contours are at 500, 1000, 1500, 2000, 3000, $4500,6000,8000$, and $10000 \mathrm{MJy} \mathrm{sr}^{-1}$. The W30 complex and SNR G11.2-0.3 along with the detected point sources have been removed. For the purpose of display, the data have been smoothed using an adaptive-filtering algorithm by selecting an area that contains 50 counts. Thus, the resolution is variable depending on the exposure and the total counts per pixel. The gray scale ranges from 0 to $700 \times 10^{-6}$ counts s$^{-1} \operatorname{arcmin}^{-2}$ for panel $(a)$, and 0 to $1300 \times 10^{-6}$ counts s$^{-1}$ arcmin ${ }^{-2}$ for panel $(b)$.

PARK et al. (see 476, L78) 


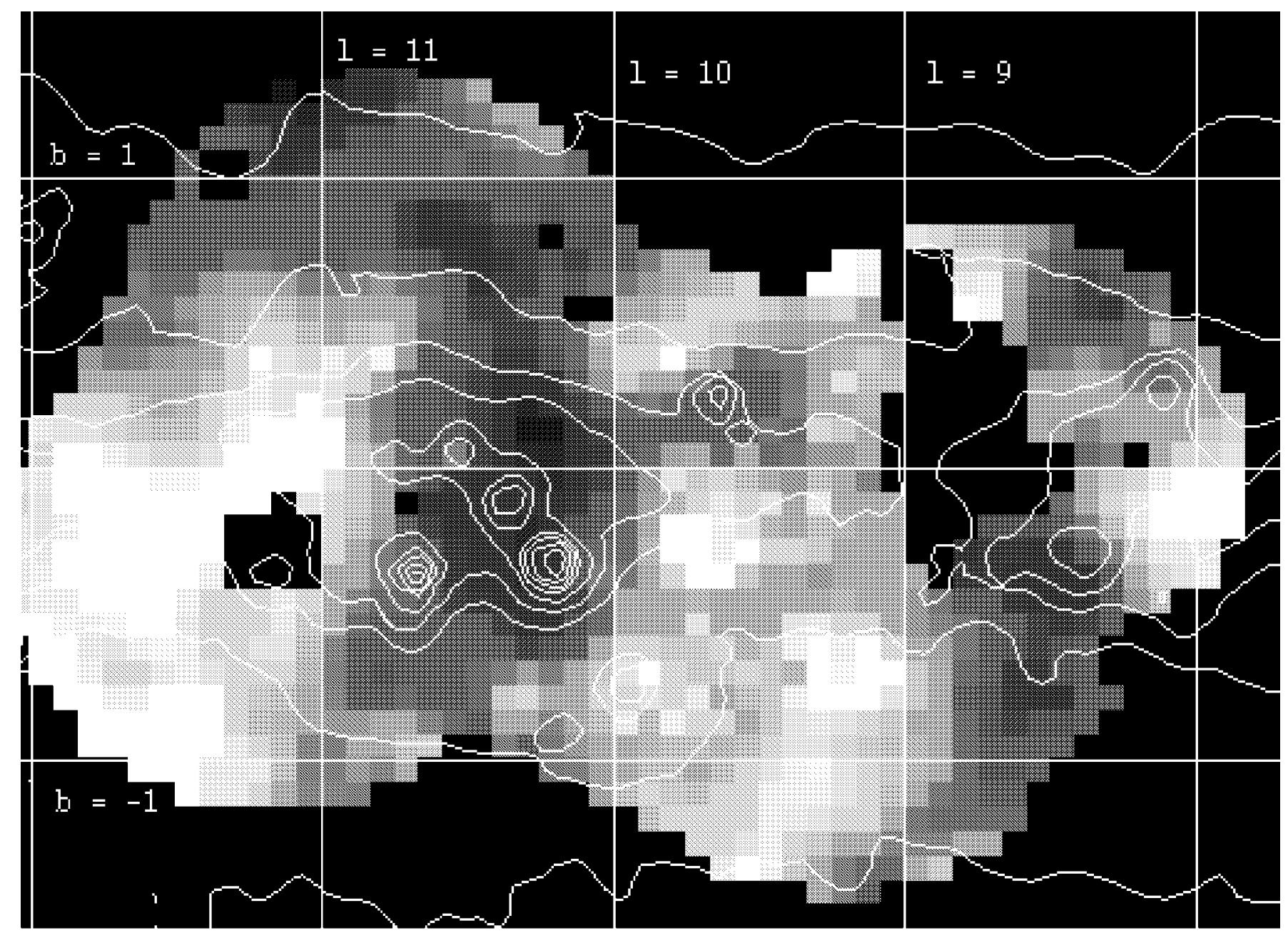

FIG. $1 b$

PARK et al. (see 476, L78) 